\title{
Allosteric alterations in the androgen receptor and activity in prostate cancer
}

\author{
Takuma Uo', Stephen R Plymate1,2 and Cynthia C Sprenger ${ }^{1}$ \\ 'Department of Medicine, University of Washington, Seattle, Washington, USA \\ ${ }^{2}$ Geriatrics Research Education and Clinical Center, VA Puget Sound Health Care System, Seattle, Washington, USA
}

Correspondence

should be addressed

to $T$ Uo

Email

tuo@u.washington.edu

\begin{abstract}
Organisms have evolved to generate biological complexity in their proteome and transcriptome from a limited number of genes. This concept holds true for the androgen receptor, which displays a diversity of inclusion/exclusion events in its structural motifs as a mechanism of resistance to the most forefront anti-androgen therapies. More than 20 androgen receptor variants that lack various portions of ligand-binding domain have been identified in human prostate cancer (PCa) samples. Most of the variants are inactive on their own, with a few exceptions displaying constitutive activity. The full-length receptor and one or more variants can be co-expressed in the same cell under many circumstances, which raises the question of how these variants physically and functionally interact with the full-length receptor or one another in the course of PCa progression. To address this issue, in this review, we will characterize and discuss androgen receptor variants, including the novel variants discovered in the last couple of years (i) individually, (ii) with respect to their physical and functional interaction with one another and (iii) in clinical relevance. Here, we also introduce the very recent understanding of AR-Vs obtained through successful development of some AR-V-specific antibodies as well as identification of novel AR-Vs by data mining approaches.
\end{abstract}

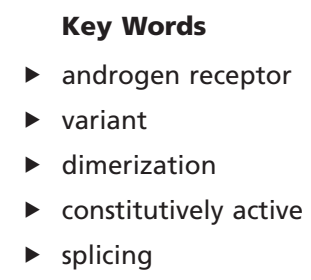

Endocrine-Related Cancer (2017) 24, R335-R348

\section{Introduction}

Since Huggins's first demonstration of surgical castration as an effective treatment for prostate cancer (PCa) in 1941, androgen deprivation therapy (ADT) has been the first-line treatment for metastatic prostate cancer (mPCa) (Huggins \& Hodges 1941, Sharifi et al. 2010, Sharifi 2015a,b). Mechanistically, ADT targets androgen dependence of the androgen receptor (AR) by lowering the availability of the androgen ligand (Mostaghel et al. 2007, 2014, Mostaghel \& Plymate 2011). ADT is initially highly effective. Nevertheless, patients with advanced PCa progress and eventually develop castration-resistant prostate cancer (CRPC) (Feldman \& Feldman 2001, de Bono et al. 2011, Fizazi et al. 2014). Throughout these clinical stages, AR is the critical driver and AR signaling is continuously activated even at the castrate level of androgen (Chen et al. 2004a, Montgomery et al. 2008, Mostaghel et al. 2011, 2014, Taplin et al. 2014, Attard et al. 2016). The mechanisms for this persistent AR activation include intratumoral androgen synthesis, overactivation of kinase signaling and increased AR expression. As a logical extension of the ADT concept, and in order to counteract continued AR signaling, most forefront 
anti-AR therapy has been developed to target ligandreceptor interactions in AR by either preventing androgen synthesis (e.g. abiraterone acetate) or antagonizing androgen binding to AR (e.g. enzalutamide). However, PCa inevitably develops resistance, even during these treatments, and progresses to lethal disease. One of the mechanisms underlying this resistance is expression of AR variants (AR-Vs) that lack various portions of the ligand-binding domain (LBD) (Dehm \& Tindall 2007, Guo et al. 2009, Sun et al. 2010, Chan et al. 2012, Li et al. 2013, Chan \& Dehm 2014, Sprenger \& Plymate 2014, Antonarakis et al. 2016, Henzler et al. 2016).

To date, numerous AR-Vs have been identified, predicted and characterized regarding their biochemical functions and clinical relevance (Hu et al. 2011, Wadosky $\&$ Koochekpour 2017). Some AR-Vs display constitutive activity and others are inactive by themselves. More complexity is added to our understanding of AR-Vs as these AR-Vs and the full-length AR (AR-FL) interact with one another to heterodimerize and exhibit unique heterodimerization-based activities, suggesting that, in many cases, a single AR-V may not account for resistance of CRPC to anti-AR therapy (Cao et al. 2014, $\mathrm{Xu}$ et al. 2015). In this review, we will characterize AR-Vs, including the novel variants discovered in the last couple of years (i) individually, (ii) with respect to their physical and functional interaction with one another and (iii) in clinical relevance.

\section{AR structure and function}

The AR represents the members of nuclear receptor class I, including estrogen receptor a (ER $\alpha)$, ER $\beta$, glucocorticoid receptor, mineralocorticoid receptor and progesterone receptor. Typically, these receptors share common regulatory mechanisms: they exist in complex with heat shock protein 90 (HSP90) and other heat shock proteins in the cytoplasm. Binding of cognate ligands frees these receptors from HSP90 followed by their translocation from cytoplasm to nucleus, where they act as sequencespecific dimerized transcription factors.

Men have one copy of the $A R$ gene at chromosome position Xq11-12 (Fig. 1). Normal splicing of canonical exons produces the transcript corresponding to AR exons $1-8$. Exon 1 encodes the entire $\mathrm{N}$-terminal domain (NTD), which represents nearly $60 \%$ of the protein and encompasses the ligand-independent activation function (AF)-1, which is subdivided into two distinct units TAU-1 and TAU-5. Exons 2 and 3 encode the DNA-binding domain (DBD). Although the $5^{\prime}$ portion of exon 4 encodes the flexible hinge region (HR), the remainder of exon 4 through exon 8 encodes the LBD, which harbors the ligand-dependent AF-2. The bipartite nuclear localization signal (NLS) is encoded by exon 3 and exon 4 (Centenera et al. 2008). Moreover, a proposed ligand-regulated nuclear export signal (NES), which operates independently of CRM1, resides in the region corresponding to the latter half of exon 5 through exon 6 (Saporita et al. 2003, Wang et al. 2004, Gong et al. 2012, Uo et al. 2017). Androgen binding to AR suppresses NES activity. The HSP90-binding site is mapped to the area corresponding to exon 5. Dimerization of AR is mediated through the dimerization box (D-box) situated in the DBD to achieve intermolecular DBD-DBD interaction and N/C-terminal interactions via the FXXLF motif in the NTD and AF-2 in the LBD (Cao et al. 2014, Xu et al. 2015). Overall, the presence of these structural motifs in $A R$ protein permits its strict androgen-dependent regulation in subcellular localization and transactivation activities.

\section{AR-Vs}

Once the structural integrity in the LBD is lost, AR is free from constraint of the LBD and in some cases becomes constitutively active and independent of the ligands (Dehm \& Tindall 2007, Libertini etal. 2007, Yang et al. 2008, Guo et al. 2009, Sun et al. 2010, Hu et al. 2011, Sprenger \& Plymate 2014) (Fig. 1). Indeed, calpain-2 cleaves AR in the HR and removes the LBD generating a constitutively active AR-V (Pelley et al. 2006, Libertini et al. 2007, Yang et al. 2008, Chen et al. 2010). On the other hand, Céraline and coworkers identified a nonsense mutation at the position of tryptophan 640 located in the HR of AR in a patient treated with anti-androgens (Ceraline et al. 2004, Jagla et al. 2007). The resulting transcript produces a truncated constitutively active variant, suggesting the presence of AR-Vs contributes to the acquisition of resistance to anti-androgen therapy. These two early observations support the evidence for the presence of ligand-independent isoforms of AR. However, since 2008, much more attention has been paid to this following the discovery of a number of AR-Vs that are missing variable portions of the LBD (Dehm et al. 2008, Guo et al. 2009, Han et al. 2017).

Initial findings were made by biased traditional molecular biology approaches, including $3^{\prime}$ rapid amplification of cDNA and targeted PCR in PCa cell lines and xenografts (Dehm et al. 2008, Hu et al. 2009). Thereafter, enormous progress was made in finding multiple species of AR-Vs owing to the advancement

Published by Bioscientifica Ltd 
A

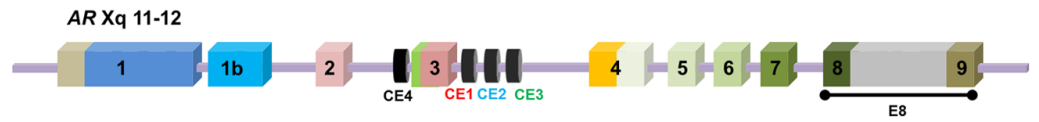

AR-FL Transcript

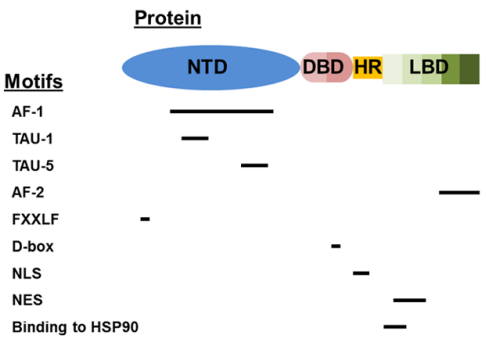

B

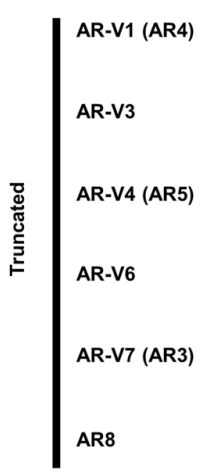

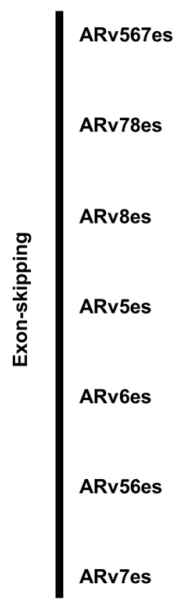

Alternative promoter AR45 $\underline{\text { Transcript }}$

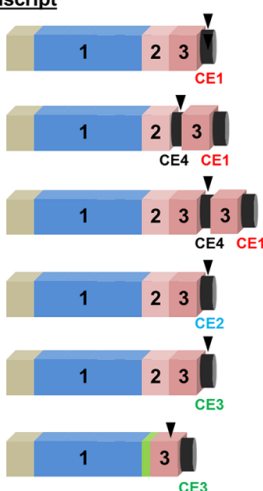

Protein
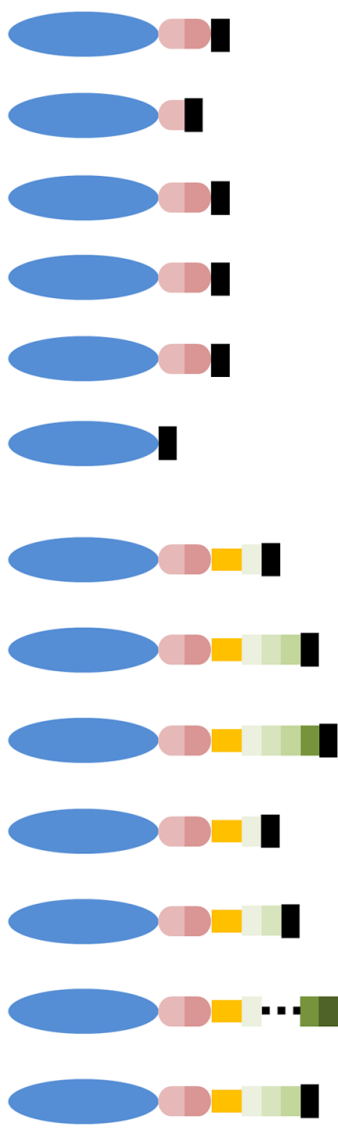

Figure 1

Schematic representation showing $A R$ gene structure of AR-FL and AR-Vs. (A) AR-FL protein (GenBank accession no. NP_000035.2) is translated from the message produced from canonical splicing to possess four major domains as well as definable functional motifs (refer to the text for details). The matched colors were used for the exons in the genome as well as transcript and the corresponding protein regions. The positions of stop codon are indicated by arrow heads. Exon 9 is defined as the exon located far downstream of exon 8. As exemplified by the transcript (GenBank accession No. NM_000044.3), the eighth exon (E8) corresponds to the unspliced long exon covering exon 8 , intron 8 and exon 9 . Furthermore, defined structural units are depicted along the protein structure of AR-FL (see text): Skipping of the specified exons leads to loss of the corresponding units. (B) The transcript and protein structures of representative AR-Vs are drawn. Truncated variants (e.g., AR-V7) are produced by incorporating CEs, whereas inclusion/exclusion of specified exons generates a variety of exon-skipping variants. Skipping the specified exons results in the frameshift, by which the respective AR-Vs have their unique C-terminal sequences (closed rectangles). Note that ARv5es, v6es, v56es and v7es are newly discovered AR-Vs through construction of splicing landscapes of $A R$ in $\mathrm{mCRPC}$. AR45 lacks the entire NTD by missing exon 1 through the use of alternative promoter. of next-generation sequencing technologies such as the establishment of integrated landscape studies of whole genome and transcriptome sequencing as well as splicing patterns in clinical samples (Robinson et al. 2015, Uo et al. 2017). Collectively, more than twenty different $A R-V s$ have been detected and identified in human samples (Marcias et al. 2010, Cao et al. 2016,
(C) 2017 Society for Endocrinology Printed in Great Britain
Coutinho et al. 2016). The mechanisms for generation of multiple $A R-V s$ appear to be varied but include the use of alternative promoters, incorporation of cryptic exons and exon-skipping events (Dehm \& Tindall 2011, Wadosky \& Koochekpour 2017).

Cumulative data have demonstrated that $A R-V s$ can be generated through selective inclusion/exclusion 
of multiple exons distributed over approximately $90 \mathrm{~kb}$ of genomic DNA. In addition to canonical exons and multiple cryptic exons located in introns 2 and 3 , the presence of exon 9, which is defined as the exon located far downstream of exon 8 , further increases the patterns of differential LBD. With limited exceptions, AR-Vs generally harbor exons 1 and 2, undergo either (i) alterative inclusion/exclusion of exon 3 in tandem with incorporation of a cryptic exon after exon 2 or 3 or (ii) alternative inclusion/exclusion of exons $4-8$ resulting in a deletion in the LBD (Robinson et al. 2015). As a definition, the former and the latter groups are termed truncated and exon-skipping variants, respectively (Uo et al. 2017).

\section{Truncated variants}

Following exons $1-3$, cryptic exons, which typically contain their own unique alternative polyadenylation sites, are included (Guo et al. 2009, Dehm \& Tindall 2011, Chan \& Dehm 2014, Ware et al. 2014) (Fig. 1). The resulting gene products contain the NTD and the DBD but are missing both the HR and the LBD, which include the motifs that favor their cytoplasmic retention, including HSP90, microtubule-binding sites and the NES. Moreover, as bipartite NLS lies in the junction of exon 3 and exon 4 , the absence of exon 4 may compromise nuclear localization of the corresponding variants. Thus, the intracellular localization of these AR-Vs determines their activities in many situations depending on the amino acid sequences stemming from cryptic exons (Hu et al. 2011). The most common AR-V, AR-V7, represents the members of this group and displays constitutive transactivation activity and includes cryptic exon 3 (CE3) (Guo et al. 2009, $\mathrm{Hu}$ et al. 2009). CE3 is able to reconstitute NLS activity thus allowing AR-V7's predominant nuclear localization (Sprenger \& Plymate 2014, Uo et al. 2017). In sharp contrast, AR-V1, $-\mathrm{V} 4$ and $-\mathrm{V} 6$ are produced as a result of inclusion of CE1, CE4, and CE2, respectively (Hu et al. 2011, Chan et al. 2012, Zhan et al. 2017). Unlike the case for CE3, inclusion of these cryptic exons fails to confer NLS activity to the corresponding AR-Vs and these variants are not found in the nucleus (Hu et al. 2011, Chan et al. 2012, Zhan et al. 2017). Furthermore, splicing of exon 2 to CE4 leads to production of AR-V3, which lacks the second zinc finger of the DBD (De Laere et al. 2017). Intriguingly, this gene product is constitutively active toward AR-responsive promoters in PCa cells (Hu et al. 2011).

More recently, drastic genomic structural rearrangements (GSRs) were discovered between exon 3 and CE3. The tumor sample in one case had a tandem duplication with a break fusion junction located between exon 3 and exon CE3 while a translocation that fuses part of chromosome 11 downstream of exon 3 was observed in another case. These GSRs appear to be a driving force to splicing of exon 3 to novel subsets of DNA sequences, which do not exist in the normal $A R$ gene. The corresponding AR-Vs contain the NTD and the DBD derived from exons 1-3 followed by novel unique peptide sequences and display constitutive activity as determined by in vitro luciferase reporter assays (Henzler et al. 2016, De Laere et al. 2017). As all of the canonical exons 1-8 appear to be present in intact forms, normal splicing may proceed to generate AR-FL even in the cells with GSRs.

In addition to nuclear AR signaling, the importance of non-genomic AR signaling has been highlighted by the presence of plasma membrane-associated AR-Vs, namely AR8, in PCa cell lines including C4-2B, 22Rv1 and CWR1-AD (Yang et al. 2011). The presence of this variant has not been reported in clinical samples yet. AR8 is encoded by the message produced as a result of the splicing of exon 1 to exon $3^{\prime}$ followed by inclusion of CE3. This variant is primarily composed of the NTD and deficient in other domains including the DBD. AR8 in complex with epidermal growth factor (EGF) receptor recruits AR-FL through FXXLF motif-mediated N/C interaction. EGF-induced activation of c-SRC promotes tyrosine phosphorylation of AR, allowing subsequent ligand-independent nuclear translocation of $\mathrm{AR}$ for continued AR signaling, even in the castrate condition.

\section{Exon-skipping variants}

Exon-skipping variants are defined as AR-Vs that undergo alternative inclusion/exclusion of canonical exons particularly exons 5-8 (Fig. 1). AR-Vs in this group commonly possess exons 1-4, which ensure the presence of full bipartite NLS, the HR, and a partial LBD (Claessens et al. 2008). ARv567es is the founding member of this group, deficient of most of the LBD, and constitutively active with predominant nuclear localization. Thus, ARv567es structurally resembles and shares most basic properties with AR-V7 (Sun et al. 2010). However, as the microtubule-binding domain of $\mathrm{AR}$ is retained, which encompasses the DBD and the HR, ARv567es but not AR-V7 undergoes taxane-sensitive trafficking to the nucleus by microtubules (Darshan et al. 2011, Carbonaro et al. 2012, Thadani-Mulero et al. 2014). The discovery of ARv567es prompted us to systematically 
and comprehensively analyze the presence of exonskipping variants in the Cancer Genome Atlas (TCGA) and Stand Up 2 Cancer (SU2C) data sets (Uo et al. 2017). AR-Vs that undergo alternative inclusion/exclusion of exons 5-8 were expressed at varying levels. Subsequently, we constructed in silico transcripts for these AR-Vs and found four novel AR-Vs that have unique features based on their predicted amino acid sequences. The LBD includes not only the ligand-binding site but also multiple regulatory sites, including HSP90-binding sites and the NES. Thus, selective exclusion of specific exons is most likely to produce unique AR-Vs with more variations, including loss of these structural motifs singly or in combination. In fact, these novel variants, namely ARv5es, v6es, v56es, and v7es, displayed their own unique characteristics, although all of them possess the impaired LBD. ARv5es, with a novel stop codon in exon 6 due to a frameshift, resembles ARv567es in that it is predominantly localized in the nucleus and is constitutively active. Interestingly, ARv5es is susceptible to the nonsense mediated decay (NMD) as we recently demonstrated in the cells that are forced to express ARv5es through CRISPER-based genomic engineering (Uo et al. 2017). NMD does occur; however, this variant, which is expressed in clinical samples from SU2C, and whose expression may be decreased by NMD, may still contribute to castration resistance. The presence of the NES, which is suppressed by androgen in AR-FL, is a dominant determinant for subcellular localization of ARv6es and ARv7es. HSP90 generally sequesters AR in the cytoplasm. The binding to HSP90 and the dominant action of the NES, due to a structurally disordered LBD, keep these two variants primarily in the cytoplasm and away from the nucleus. Thus, ARv6es and v7es are categorized as inactive AR-Vs. Notably, ARv56es is localized in the nucleus and the cytoplasm, presumably due to the absence of HSP90 binding and the NES. In spite of the absence of these two inhibitory factors, the ARv56es variant is inactive due to the presence of the intact C-terminal half of the LBD. Among the numerous exon-skipping AR-Vs, ARv5es and ARv567es stand out as constitutively active variants and potentially drive CRPC growth in patients receiving the current most forefront AR therapies, which makes them attractive as targets for the development of novel therapeutics.

\section{Other variants}

AR45 is produced through the use of an alternative promoter and incorporation of exon 1b (Ahrens-Fath et al. 2005, Hu et al. 2016). It loses its trans-activating ability and acts in a dominant-negative fashion to inhibit the function of AR-FL by forming a heterodimer. Nonsense mutations have been identified much less frequently than aforementioned variants while conceptually this can be accepted as the mechanism of generation of constitutively active variants.

\section{Heterodimerization}

It has been long known that AR-Vs and AR-FL are simultaneously expressed in a single cell in cultured PCa cells (Liu et al. 2014). It remains to be determined whether these observations obtained from overexpression studies can be reproduced in clinical specimens with endogenous levels. Cellular heterogeneities in tumor samples have made it inherently difficult to examine whether any given detected forms of AR are co-expressed in the same cell. Nevertheless, it has now become possible to analyze variations in expression and splicing patterns of any given gene, such as AR, at single-cell resolution due to advancement in technologies and techniques of single cell isolation and downstream analysis (Miyamoto et al. 2014, 2015). The mechanisms by which multiple forms of AR are simultaneously produced include: alternative splicing alone or in couple with gene rearrangement or gene amplification plus point mutations. As we have already mentioned AR8 and AR45, this section will be limited to AR-Vs harboring both the NTD and the DBD.

Most AR-Vs contain exons 1-3 and thus they have the FXXLF motif and the DBD, which are independently necessary for dimerization of AR. When AR-Vs are co-expressed in the same cell, different forms of AR appear to physically interact with one another leading to functional outcomes (Cao et al. 2014, Sartor \& Dong 2015, Xu et al. 2015, Zhan et al. 2017). For example, constitutively active AR-Vs, AR-V7 and ARv567es, can homodimerize but can also heterodimerize with AR-FL in an androgenindependent manner. The heterodimerization is reportedly necessary to drive canonical AR signaling during androgen-directed therapy (Cao et al. 2014, Xu et al. 2015). Interestingly AR-V1, which is mainly cytoplasmic and displays minimum transactivation activities due to a lack of a defined NLS, can also activate AR-FL in an androgenindependent manner while displaying a dominantnegative action on AR-V7 (Zhan et al. 2017). On the other hand, two other cytoplasmic truncated AR-Vs, AR-V4 and $-\mathrm{V} 6$, are translocated into nucleus when they co-exist with either AR-V7 or liganded AR-FL. Nuclear localization of these variants display transactivation activities and

Published by Bioscientifica Ltd. 
confer castration-resistant cell growth (Zhan et al. 2017). Therefore, heterodimerization-based activities of AR-Vs, which are otherwise inactive, add another layer of complexity to AR-V signaling.

In addition to alternative splicing, other cellular milieus account for the mechanisms of co-expression of differential forms of AR in the same cell. Genomic amplification of the AR locus is one of the most common events in CRPC and point mutations may occur in one of the resulting multiple copies of AR (Azad et al. 2015, Romanel et al. 2015, Henzler et al. 2016). Under such a condition, AR-Vs derived from a mutant gene can be simultaneously expressed with AR-FL. A novel mutation $(3465 \mathrm{C}->\mathrm{T})$ in exon 6 of the AR gene was discovered from the sample of a patient who relapsed after ADT (Han et al. 2017). This is a nonsense mutation resulting in potential production of AR-Q784* (in which the codon for Q784 is converted to a stop codon), which is mainly localized in the nucleus but is transcriptionally inactive. Experimentally, AR-Q784* was able to heterodimerize with and transactivate AR-FL even in the absence of androgen (Han et al. 2017). This may occur as gene amplifications of AR are common in CRPC samples. When a point mutation corresponding to the Q784* mutant is introduced, AR-Q784* and AR-FL may be co-expressed in the same cell. This heterodimerization appears to underlie the mechanisms by which this particular CRPC acquired resistance to ADT, although we have to wait for the rigorous demonstration of the co-occurrence of AR-Q784* and AR-FL in the same cell. These observations warrant a further investigation of these heterodimerization-based activities in relation to exon-skipping variants, most of which are transcriptionally inactive on their own.

\section{The mechanisms and regulation of formation of AR-Vs}

\section{Truncated AR-Vs}

The mechanisms by which the AR-Vs arise and the regulation of their formation are complex and not completely understood. In the case of the truncated variants such as $A R-V 1, A R-V 3$ and $A R-V 7$, it is generally thought that they are produced as the result of aberrant splicing of pre-mRNA instigated by cell stress, most commonly a decrease in intratumoral androgens, and less likely to arise due to GSRs of the $A R$ gene (Dehm \& Tindall 2007, 2011, Dehm et al. 2008, Dehm 2013a,b, Liu et al. 2014, Henzler et al. 2016). Typically, truncated variants like these harbor the canonical exon 1 through exon 3 followed by incorporation of cryptic exons (CE1, 2, 3 and 5 ) in intron 3 , each of which carries the polyadenylation signals allowing early termination of $A R$ transcription as well as stabilization of the corresponding message. Previously we demonstrated that ADT conditions are causally related to RNA splicing to generate $A R-V 7$ production. Specifically, several RNA splicing factors were more efficiently recruited to the 3 ' splicing site for $A R-V 7$ (3'ss next to CE3) but not 3'ss next to exon 4 under ADT conditions compared to non-ADT conditions (Liu et al. 2014). This aberrant splicing activity was also generated by the addition of enzalutamide. When androgens were replaced in the system, the splicing proteins were no longer attracted to the splicing enhancers and canonical $A R$ transcripts were generated. We identified two RNA splicing enhancers and their binding proteins, U2AF65 and ASF/SF2, which had critical roles in splicing $A R$ premRNA into $A R-V 7$. This notion was corroborated by the results from a subsequent study demonstrating that in animal models bearing castration-resistant, enzalutamideresistant xenografts, treatment with bromodomain inhibitors decreased $A R-V 7$ production in association with a decrease in U2AF65 and ASF/SF2 mRNA (Asangani et al. 2016). Several studies have also demonstrated that both c-MYC and NF-kappaB2/p52 increase $A R$-V7 (Nadiminty \& Gao 2012, Nadiminty et al. 2013, 2015, Liu et al. 2016, Wang et al. 2016a). This activity is mediated by the splice factor protein hnRNPA1. Other investigators have shown that miRNAs may also induce splicing to $A R-V 7$. All the data in which AR-truncated variants are generated by splicing of $A R$ pre-mRNA have been in cell lines or tumors in which there is an increased copy number of the androgen receptor gene (Yang et al. 2016). Another mechanism by which $A R-V 7$ may arise is due to structural rearrangements in the $A R$ gene. Dehm and coworkers originally introduced this concept when they described a structural rearrangement in introns 1, 2 and 3 of the $A R$ gene present in the castrate-resistant PCa cell line 22Rv1, which was associated with increased expression of $A R-V 7$ (Dehm et al. 2008). Recently, De Laere and coworkers examined the presence of GSRs of the $A R$ gene in freecirculating tumor DNA in men with advanced PCa/CRPC (De Laere et al. 2017). Out of 33 plasma samples, 17 samples (15 patients) displayed intra-AR GSRs. Four major types of GSRs were detected: deletions, inversions, tandem duplications and translocations. Further studies were then conducted on 17 of those patients in whom samples were collected at baseline, the time of initiation 
of second-line endocrine therapy. $\sim 24 \%$ of the patients $(4 / 17)$ had GSRs and all of these were in men who progressed after less than 6 months on therapy; no GSRs were detected in men who were on abiraterone or enzalutamide for $>6$ months. A further nine samples were examined at the time of progression and demonstrated that almost $90 \%(8 / 9)$ of the men who progressed in less than 6 months had $A R$ GSRs detected in their free DNA, while only 1 out 3 men who progressed after 6-12 months on therapy did. In those patients who had GSRs, the correlation between the presence of GSRs and the detection of AR-V mRNA in these men's circulating tumor cells (CTCs) was high, 11 out of 13 samples (10/11 patients). The most commonly found $A R-V$ transcripts were $A R-V 7$ and $A R-V 3$, and only the transcripts for seven truncated $A R-V s$, not exon-skipping variants, were included in the analysis. In addition, the majority of CTCs expressed more than one $A R-V$. Interestingly, only 10 of the 15 patients with $A R-V$ mRNA had structural rearrangements of the $A R$ gene, as determined by whole genome sequencing. However, 4 out the 5 men without GSRs did have $A R$ amplification, suggesting that truncated AR-Vs may arise through multiple mechanisms. Dehm's and De Laere's studies demonstrated that as the $A R$ gene is more deeply sequenced for GSRs, the greater the likelihood of finding patients who express GSRs associated with AR-Vs (Henzler et al. 2016, De Laere et al. 2017). Moreover, as mentioned above in regard to the newly discovered GSRs between exon 3 and CE3, we may see a number of previously unappreciated types of truncated AR-Vs in the future. Nevertheless, except for those cases in which the rearrangement has been shown to directly lead to AR-V expression, it has yet to be determined whether the GSRs truly are responsible for generating AR-Vs and are responsible for resistance to AR-directed therapies and shortened overall survival. Overall, the formation of truncated AR-Vs appears to be due mostly to aberrant splicing.

\section{Exon skipping AR-Vs}

The aforementioned genomic structural rearrangements of $A R$ most commonly account for generation of exon skipping variants, particularly ARv567es. Initially, two distinct GSRs were reported to be associated with generation of ARv567es expression in the LuCaP-series of human prostate xenografts. LuCaP 86.2 is heterogeneous with at least two stable CRPC subclones; in view of $A R$ GSR, one clone maintains normal $A R$ gene structure and does not express ARv567es, while the other harbors an intragenic deletion of $A R$ exons 5-7, leading to expression of ARv567es (Fig. 2). On the other hand, in the human xenograft LuCaP 136 a GSR was found that resulted in an inversion of exons 5, 6 and 7 in the $A R$ gene resulting in ARv567es (Fig. 2) (Li et al. 2013, Nyquist \& Dehm 2013, Isharwal et al. 2017). Of particular interest, after six passages in non-castrate mice, LuCaP 136 no longer expressed ARv567es nor could the inversion be detected. The xenograft, which was originally castrate resistant, became castration sensitive, thus strongly suggesting that it was the presence of constitutively active ARv567es that led to castration resistance and that in the presence of androgen the constitutively active variants are not dominant. In a recent study, Henzler and coworkers reported on a third $A R$ GSR associated with ARv567es. It was detected in multiple metastases from a single patient who died following nine years of ADT (Henzler et al. 2016). The GSR was a complex

\section{$A R \times q 11-12$}

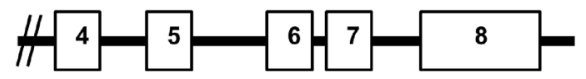

\section{LuCaP 136}

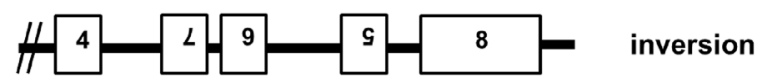

\section{LuCaP 86.2}

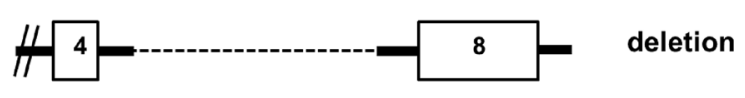

$A R \times q 11-12$

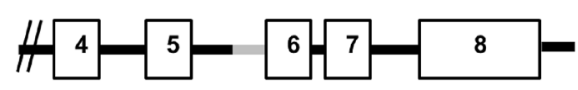

patient C-6

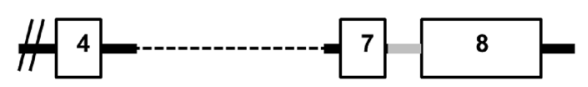

duplication deletion

\section{Figure 2}

Three distinct $A R$ GSR driving to synthesis of ARv567es. 9-kb inversion and deletion $A R$ exons 5-7 were detected in human xenografts LuCaP 136 and LuCaP 86.2, respectively. The cells undergoing these GSR exclusively express ARv567es. mPCa cells in the rapid autopsy patient C-6 have the unique AR GSR after a multi-step rearrangement (i.e., combined duplication and deletion events), leading to simultaneous deletion of $A R$ exons 5 and 6 . The resulting $A R$ gene has the newly structured intron 7 , which is mostly composed of the portion of original intron 5 (gray line). Owing to the presence of intact exon 7, splicing of exon 4-8 to skip exon 7 appears to be the prerequisite step to produce ARv567es in this situation.
() 2017 Society for Endocrinology Printed in Great Britain
Published by Bioscientifica Ltd. 
arrangement of three separate rearrangements occurring on the same allele that resulted in generation of ARv567es (Fig. 2). The rearrangement was present in tissue from all five metastatic sites for which genomic DNA was available for sequencing; the genomic sequencing covered between 83 and 89\% of the AR. Expression of ARv567es protein was detected by immunohistochemistry with an ARv567esspecific antibody. This group further demonstrated that when multiple metastases were examined from 15 patients, 6 of the 15 patients (10/30 metastases) harbored GSRs and 7 of these metastases demonstrated more than one GSR (Henzler et al. 2016). Although all tumors were derived from patients that were castrate resistant, not all GSRs could be shown to result in a constitutively active AR-V. Additionally, no tumor that expressed AR-V7 had a GSR but all had increased $A R$ copy number. Of further note, we also examined 19 primary tumor samples from patients who were not castrate and no GSRs were detected. However, with current $A R$ sequencing, approximately $65 \%$ of the $A R$ gene is covered. As a greater proportion of the $A R$ gene is interrogated to a greater depth, it may be that more $A R$ GSRs will be uncovered. In summary, multiple mechanisms may be responsible for AR-V formation; however, whether the mechanism is AR GSR or splicing or a combination has yet to be determined.

\section{Expression of multiple AR-Vs}

In clinical data, such as that from SU2C, Uo and coworkers and more recently De Laere and coworkers have shown that rarely do tumors express a single $A R-V$, more commonly they express between four and ten AR-Vs per tumor (Robinson et al. 2015, De Laere et al. 2017, Uo et al. 2017). Furthermore, recent single CTC analysis revealed that one-sixth of the CTCs co-expressed more than one $A R-V$. However, only a few of these AR-Vs have been shown to be constitutively active. Such evidence further confounds the picture as to whether or not the variants that are associated with resistance are generated at random from the increased $A R$ copy number seen in CRPC, indicating that the basic castration resistance mechanism is the increase in $A R$ gene expression as originally suggested by Chen and Sawyers (Chen et al. 2004b).

\section{AR-V transcriptome}

Multiple studies have examined the AR-V transcriptome in human tissues and cell lines. Hornberg and coworkers used microarray data to examine gene expression in patients presenting with bone metastases (Hornberg et al. 2011). When they examined gene expression in those tumors that expressed $A R-V 7$ or ARv567es mRNA, they demonstrated that the AR-V tumors had an upregulation in genes associated with progression through the cell cycle e.g. UBE2C and CDC25. In addition, UGT2B15 and 17 were also increased. These two glucuronidating genes enhance glucuronidation of androgens and would perpetuate the decrease in intracellular androgens thereby potentially enhancing AR-V generation. A second study by $\mathrm{Hu}$ and coworkers examined the AR-V transcriptome by either blocking AR-FL activity in LNCaP 95 and VCaP cells that expressed both AR-V7 and AR-FL or by expressing ARv567es in LNCaP APIPC cells, a derivative of LNCaP cells that have been engineered to no longer express the endogenous $\mathrm{AR}$ and were subsequently transfected with an ARv567es expression vector (Hu et al. 2012). Gene expression arrays again demonstrated increased expression of cell cycle proliferation genes as well as UGT2B17 in each cell line when AR-FL receptor was either blocked, in the case of LNCaP95 and APIPC cells, or removed, as in the APIPC cells. Canonical AR genes such as KLK3, TMPRSS2 and FKBP5 were also expressed in response to AR-Vs. However, not all of the reported transcriptomes for AR-Vs are identical. In part, this may be expected, due to epigenetic differences between cell types and culture conditions. It may also be due to differences between the variants themselves. For instance, ARv567es retains the HR of exon 4, which maintains the classic AR nuclear localization sequence and has been shown to have different nuclear export and import properties. The HR, however, also contains an acetylation motif that could alter chromatin binding. As AR-V7 and AR-V3 do not contain the HR, there is the likelihood of differences between variant's activities.

\section{AR-V cistrome}

A clear definition of the AR-V cistrome has been hampered by the lack of specific AR-V antibodies for ChIP-seq precipitation. Therefore, specific cistromic data from the variants has been difficult to obtain since most data have been derived from negative selection for AR-V or by generating artificial cell constructs and performing ChIP-seq with an N-terminal AR antibody. The first cistrome to be described was by $\mathrm{Lu}$ and coworkers for 22Rv1 cells in which they used shRNA methods to eliminate AR-FL expression and then used an AR-Nterminal antibody to perform ChIP-seq assays (Lu et al. 2015). In the $22 \mathrm{Rv} 1$ cistrome, they found specific AR-V7binding sites as well as cell cycle genes. In addition, they found canonical AR-binding sites. Of importance, their

Published by Bioscientifica Ltd 
data demonstrated the presence of potentially unique AR-V7-binding sites. However, as Marcias and coworkers have reported, cells expressing AR-V7 can also contain several AR-Vs, each of which has an AR-N-terminus that could bind the N-terminal antibody, and thus, lead to interfering data (Marcias et al. 2010). In a second paper, Chan and coworkers used the CWR-AD1-derived cell line (R1-D567) harboring an engineered genomic deletion of AR exons $5-7$ by modeling the AR gene rearrangement in LuCaP86.2. AR-FL and ARv567es are predominantly and exclusively expressed in parental CWR-AD1 and R1-D567 cell lines, respectively (Chan et al. 2015). Thus, an N-terminal antibody can be used in each cell line to uncover the cistromes of either AR-FL or ARv567es. Their data did not demonstrate the presence of any unique AR-V-binding sites but rather showed a cistrome similar to a ligand-bound AR-FL canonical cistrome, albeit with somewhat weaker ARv567es binding to androgen response elements (AREs) than liganded AR-FL. It should be noted that neither of these studies used AR-V-specific antibodies and although both were done on a CWR-1 backbone one addressed AR-FL and the other addressed ARv567es. A further factor that has yet to be addressed in the ChIP-seq studies is the heterodimerization between AR-Vs and AR-FL receptors in the absence of androgen, as described by $\mathrm{CaO}$ and coworkers and $\mathrm{Xu}$ and coworkers (Cao et al. 2014, Xu et al. 2015). Those papers provide evidence that the constitutively active AR-Vs heterodimerize between their DBDs, translocate to the nucleus in the absence of ligand and bind to chromatin and canonical AREs. However, only AR-V7 bound to the UBE2C enhancer region, further supporting the idea that UBE2C expression is AR-V regulated. Recently, Zhan and coworkers also demonstrated that AR-Vs that are not transported to the nucleus can heterodimerize with constitutively active variants or liganded AR-FL, translocate to the nucleus, and then generate a canonical or variant-specific signal depending on the binding partner (Zhan et al. 2017). All these combinations of generating an AR-V transcriptome or cistrome can lead to a complex array of AR signaling mechanisms in AR-driven CRPC. This complexity is especially true since a single tumor and potentially a single cancer cell can contain multiple AR-Vs.

\section{Clinical relevance}

Multiple in vitro studies have been performed to demonstrate that AR-Vs that are constitutively active and have lost all or part of the LBD are resistant to first- and second-generation AR inhibitors, including AR ligand inhibitors such as enzalutamide and the CYP17 inhibitor abiraterone (Dehm \& Tindall 2011, Hu et al. 2012, Dehm 2013a, Chan \& Dehm 2014, Sprenger \& Plymate 2014, Antonarakis et al. 2016). When PCa xenografts express these constitutively active receptors, either endogenously or are engineered to express AR-Vs, they are resistant to both androgen deprivation strategies as well as secondgeneration anti-androgens. These studies provide evidence that the variants can drive a tumor and do not respond to LBD inhibition, as would be predicted based on their lack of a LBD. However, because the $A R-V$ mRNA is expressed at very low levels in castrate-resistant tumors, it had been questioned as to whether their expression was relevant. However, Robinson and coworkers demonstrated in the SU2C data, that the incidence of multiple $A R-V s$ increased significantly with castration resistance, especially when compared to primary tumors (Robinson et al. 2015). Arguably, the first data to clinically demonstrate that the expression of $A R-V 7$ was associated with development of resistance to anti-androgen therapy was that from the Anatonarakis and Luo labs in which they showed that the detection of $A R-V 7$ transcripts in CTCs predicted both primary and secondary resistance to abiraterone or enzalutamide; in addition, they found that $A R-V 7$ was present in greater than $40 \%$ of men who were resistant to these drugs (Antonarakis et al. 2014, 2016). Their studies have been generally replicated by multiple techniques in various laboratories (Scher et al. 2016). However, expression in tumors of mRNA relative to AR-FL was still generally small, $<1 \%$, and still begged the question as to whether the variant was a marker of resistance or actually causative. With the development of relatively specific antibodies to the AR-V7 protein, it became apparent that AR-V7 protein was present in the nucleus of clinical PCa metastases and increased as the tumors became resistant to second-line anti-androgens. This finding was important because it demonstrated that a protein that functions as a transcription factor was observed in the nucleus of castration-resistant patients at levels that could be detected by IHC and thus the question as to whether the message level was higher or lower than AR-FL message becomes mute. Furthermore, Scher and coworkers have demonstrated that AR-V7 protein was also present in CTCs of patients resistant to enzalutamide or abiraterone (Scher et al. 2016). However, it should be noted that the number of patients with AR-V7 protein in their CTCs was significantly lower than those with detectable $A R-V 7$ mRNA. Of particular interest is that 
Scher and coworkers also found that those patients who are positive for AR-V7 protein had a better response to taxanes. In this and other studies, when metastatic tissue was examined in the same patient during conversion from castration-sensitive to the castration-resistant state, AR-V7 protein increased significantly (Scher et al. 2016, Welti et al. 2016). Furthermore, as had originally been suggested by Hornberg and coworkers, patients expressing $A R-V 7$ had a significantly shortened overall survival than those in the lower quartile of $A R-V 7$ expression by approximately 4 months. This would be consistent with AR- $V$ transcriptomes that demonstrated higher level of proliferative genes. As mentioned previously, of recent interest is a paper from Yan Dong's laboratory in which she demonstrated that when truncated AR-Vs that are not constitutively active on their own hybridize through the DBD with unliganded AR-V7 or liganded AR, they cause nuclear translocation of the heterodimer, activate a variant specific luciferase reporter, have enhanced growth in androgen deprivation conditions in vitro and decrease the response to enzalutamide (Zhan et al. 2017). On the other hand, there has been a long-standing belief that constitutive active AR-Vs also require AR-FL to exhibit their resistance in some situations (Watson et al. 2010). Indeed, co-expression of AR-FL and AR-Vs is well documented in PCa cells lines and human specimens (Miyamoto et al. 2015). It is well reasoned to investigate how AR-FL contributes to activities to AR-Vs as this knowledge is crucial to understand how to best target AR-V signaling in CRPC patients. Thus, these new data by Zhan and coworkers would expand the role of AR-Vs such that they do not have to be constitutively active in order to generate castration resistance.

\section{Therapy}

Since the greatest volume of clinical data and potential effects on outcomes has been generated with AR-V7, efforts have been made to inhibit AR-V7. At the present time, most of the data presented in the literature confirm that AR-V7 is a biomarker; however, until it can be specifically inhibited, its role in driving PCa clinically cannot be confirmed. Therapies aimed toward inhibition of constitutively active AR-Vs have taken four general approaches: (1) inhibition of AR N-terminal activity, (2) degradation of AR-V7, (3) inhibition of splicing and (4) inhibition of the binding at the AR-DBD.

Inhibition of AR N-terminal activity has been addressed using a number of compounds, although at the present time EPI-506 is the only compound currently in clinical trial (phase 1) (Myung et al. 2013, Yang et al. 2016). EPI- 506 and its precursors EPI-001 and EPI-002 are derivatives of bisphenol A. These compounds have been shown to directly bind to the TAU-5 in the N-terminus of the AR and inhibit AR-FL as well as AR-V7 and ARv567es signaling and growth. Pre-clinical studies with this class of compounds have shown efficacy in vitro as well as in human xenograft animal models. Phase 1 clinical trials are currently in process.

Several compounds have been developed that enhance AR-V7 ubiquitination and proteasome degradation of AR-V7 including niclosamide, ASC-J9 and galeterone. Niclosamide is an anthelmintic and has been given to humans, but only for short periods of time, and whether effective blood levels can be reached in humans without toxicity has not been determined (Nadiminty \& Gao 2012, Nadiminty et al. 2013, 2015). ASC-J9 is effective in vitro and in vivo in xenograft models but has not been tested in PCa trials (Yamashita et al. 2012, Wang et al. 2016b, Wen et al. 2016). Galeterone has recently been used in a large phase 2 clinical trial in which $A R-V 7$ expression in CTCs was a requirement for entry into the trial. In pre-clinical studies, galeterone degraded AR-V7 but not AR-FL. The trial was not successful because galeterone, which is also a CYP17 inhibitor, showed no better results than abiraterone in the comparator arm of the trial (Njar \& Brodie 2015). In addition, its effects on AR-V7 expression in these trial patients are yet to be reported.

Inhibition of splicing from $A R-F L$ is an attractive target but few viable compounds are available. JQ1, a bromodomain 4 inhibitor, has been shown to suppress variant expressing CRPC xenografts in association with a decrease in the splicing proteins responsible for splicing of $A R-F L$ pre-mRNA to $A R-V 7$. Although the growth of the xenografts slowed significantly, JQ1 has multiple effects on cells and the androgen receptor; thus, the decrease in $A R$-V7 splicing in this model may not be the predominant mechanism of decreased growth (Asangani et al. 2016).

While targeting of the N-terminus is difficult due to the intrinsic disorder of this domain, targeting the highly conserved DBD region, which is present in both AR-FL and AR-Vs, is gaining new ground. $\mathrm{X}$-ray crystallography of the AR-DBD in complex with co-proteins and DNA has provided insights into how to disrupt this binding. Dalal and coworkers have recently demonstrated that targeting the DBD of the AR is effective in inhibiting AR-FL and AR-Vs in vitro and 
in xenografts (Dalal et al. 2014, Li et al. 2014). Smallmolecule inhibitors were designed to specifically block AR binding to DNA at the DBD domain and do not affect the LBD. These molecules abolished AR transcriptional activity when tested on LNCaPs, PC3 cells transiently expressing either AR-FL or AR-Vs or in CWR-R1 cells genetically modified to express AR-Vs. ChIP studies further verified that these small-molecule inhibitors blocked DNA binding. In vivo studies using LNCaP demonstrated decreased tumor growth in treated mice. In another study, Lim and coworkers demonstrated that pyrvinium pamoate (PP) inhibits AR activity in LNCaP and LAPC4 cells via the DBD. Further, PP slowed tumor growth in mice bearing 22Rv1 xenografts, which express AR-FL and AR-V7. These studies support the idea that targeting domains common to both AR-FL and AR-Vs, such as the DBD, has therapeutic potential. Human studies have not been performed yet.

\section{Summary}

The full-length $\mathrm{AR}$ is a classical nuclear hormone receptor that is dependent upon binding of ligand for activation. Current anti-androgen therapies, however, seem to drive the expression of diverse AR-Vs, some of which are constitutively active, and therefore, no longer require ligand for activation. The exact mechanisms by which these AR-Vs arise are not known, but various studies support both alternative splicing and genomic rearrangements as possible mechanisms. A better understanding of AR-Vs in CRPC is relevant not only to gaining insights into the development of resistance to current AR-directed therapies but importantly to further developing an understanding of how the AR continues to be the driver for most CRPC. Expanding our knowledge of AR-driven PCa will provide new nodes that can then be targeted for the development of 'precision' therapy for AR-driven CRPC.

\section{Declaration of interest}

T U and C C S declare that there is no conflict of interest that could be perceived as prejudicing the impartiality of the research reported. S R P is a consultant for ESSA Pharma, Inc.

\section{Funding}

This work was supported by the Department of Defense (W81XWH-12PCRP-TIA), Veterans Affairs Research Program and National Cancer of Institute (P01CA163227, P50 CA 097186).

\section{References}

Ahrens-Fath I, Politz O, Geserick C \& Haendler B 2005 Androgen receptor function is modulated by the tissue-specific AR45 variant. FEBS Journal 272 74-84. (doi:10.1111/j.1432-1033.2004.04395.x)

Antonarakis ES, Lu C, Wang H, Luber B, Nakazawa M, Roeser JC, Chen Y, Mohammad TA, Fedor HL, Lotan TL, et al. 2014 AR-V7 and resistance to enzalutamide and abiraterone in prostate cancer. New England Journal of Medicine 371 1028-1038. (doi:10.1056/ NEJMoa1315815)

Antonarakis ES, Armstrong AJ, Dehm SM \& Luo J 2016 Androgen receptor variant-driven prostate cancer: clinical implications and therapeutic targeting. Prostate Cancer and Prostatic Diseases 19 231-241. (doi:10.1038/pcan.2016.17)

Asangani IA, Wilder-Romans K, Dommeti VL, Krishnamurthy PM, Ape IJ, Escara-Wilke J, Plymate SR, Navone NM, Wang S, Feng FY, et al. 2016 BET bromodomain inhibitors enhance efficacy and disrupt resistance to $\mathrm{AR}$ antagonists in the treatment of prostate cancer. Molecular Cancer Research 14 324-331. (doi:10.1158/1541-7786.MCR15-0472)

Attard G, Parker C, Eeles RA, Schroder F, Tomlins SA, Tannock I, Drake CG \& de Bono JS 2016 Prostate cancer. Lancet 387 70-82. (doi:10.1016/S0140-6736(14)61947-4)

Azad AA, Volik SV, Wyatt AW, Haegert A, Le Bihan S, Bell RH, Anderson SA, McConeghy B, Shukin R, Bazov J, et al. 2015 Androgen receptor gene aberrations in circulating cell-free DNA: biomarkers of therapeutic resistance in castration-resistant prostate cancer. Clinical Cancer Research 21 2315-2324. (doi:10.1158/1078-0432.CCR-142666)

Cao B, Qi Y, Zhang G, Xu D, Zhan Y, Alvarez X, Guo Z, Fu X, Plymate SR, Sartor O, et al. 2014 Androgen receptor splice variants activating the full-length receptor in mediating resistance to androgen-directed therapy. Oncotarget 5 1646-1656. (doi:10.18632/oncotarget.1802)

Cao S, Zhan Y \& Dong Y 2016 Emerging data on androgen receptor splice variants in prostate cancer. Endocrine-Related Cancer 23 T199-T210. (doi:10.1530/ERC-16-0298)

Carbonaro M, Escuin D, O'Brate A, Thadani-Mulero M \& Giannakakou P 2012 Microtubules regulate hypoxia-inducible factor-1alpha protein trafficking and activity: implications for taxane therapy. Journal of Biological Chemistry 287 11859-11869. (doi:10.1074/jbc. M112.345587)

Centenera MM, Harris JM, Tilley WD \& Butler LM 2008 The contribution of different androgen receptor domains to receptor dimerization and signaling. Molecular Endocrinology 22 2373-2382. (doi:10.1210/me.2008-0017)

Ceraline J, Cruchant MD, Erdmann E, Erbs P, Kurtz JE, Duclos B, Jacqmin D, Chopin D \& Bergerat JP 2004 Constitutive activation of the androgen receptor by a point mutation in the hinge region: a new mechanism for androgen-independent growth in prostate cancer. International Journal of Cancer 108 152-157. (doi:10.1002/ ijc.11404)

Chan SC \& Dehm SM 2014 Constitutive activity of the androgen receptor. Advances in Pharmacology 70 327-366.

Chan SC, Li Y \& Dehm SM 2012 Androgen receptor splice variants activate androgen receptor target genes and support aberrant prostate cancer cell growth independent of canonical androgen receptor nuclear localization signal. Journal of Biological Chemistry 287 19736-19749. (doi:10.1074/jbc.M112.352930)

Chan SC, Selth LA, Li Y, Nyquist MD, Miao L, Bradner JE, Raj GV, Tilley WD \& Dehm SM 2015 Targeting chromatin binding regulation of constitutively active AR variants to overcome prostate cancer resistance to endocrine-based therapies. Nucleic Acids Research 43 5880-5897. (doi:10.1093/nar/gkv262)

Chen C, Welsbie D, Tran C, Baek S, Chen R, Vessella R, Rosenfeld M \& Sawyers C 2004a Molecular determinants of resistance to http://erc.endocrinology-journals.org

DOI: $10.1530 /$ ERC-17-0108
(C) 2017 Society for Endocrinology Printed in Great Britain
Published by Bioscientifica Ltd 
antiandrogen therapy. Nature Medicine 10 33-39. (doi:10.1038/ nm972)

Chen CD, Welsbie DS, Tran C, Baek SH, Chen R, Vessella R, Rosenfeld MG \& Sawyers CL $2004 b$ Molecular determinants of resistance to antiandrogen therapy. Nature Medicine 10 33-39. (doi:10.1038/ nm972)

Chen H, Libertini SJ, Wang Y, Kung HJ, Ghosh P \& Mudryj M 2010 ERK regulates calpain 2-induced androgen receptor proteolysis in CWR22 relapsed prostate tumor cell lines. Journal of Biological Chemistry $\mathbf{2 8 5}$ 2368-2374. (doi:10.1074/jbc.M109.049379)

Claessens F, Denayer S, Van Tilborgh N, Kerkhofs S, Helsen C \& Haelens A 2008 Diverse roles of androgen receptor (AR) domains in AR-mediated signaling. Nuclear Receptor Signaling 6 e008.

Coutinho I, Day TK, Tilley WD \& Selth LA 2016 Androgen receptor signaling in castration-resistant prostate cancer: a lesson in persistence. Endocrine-Related Cancer 23 T179-T197. (doi:10.1530/ ERC-16-0422)

Dalal K, Roshan-Moniri M, Sharma A, Li H, Ban F, Hassona MD, Hsing M, Singh K, LeBlanc E, Dehm S, et al. 2014 Selectively targeting the DNA-binding domain of the androgen receptor as a prospective therapy for prostate cancer. Journal of Biological Chemistry $\mathbf{2 8 9}$ 26417-26429. (doi:10.1074/jbc.M114.553818)

Darshan MS, Loftus MS, Thadani-Mulero M, Levy BP, Escuin D, Zhou XK, Gjyrezi A, Chanel-Vos C, Shen R, Tagawa ST, et al. 2011 Taxaneinduced blockade to nuclear accumulation of the androgen receptor predicts clinical responses in metastatic prostate cancer. Cancer Research 71 6019-6029. (doi:10.1158/0008-5472.CAN-11-1417)

de Bono JS, Logothetis CJ, Molina A, Fizazi K, North S, Chu L, Chi KN, Jones RJ, Goodman OB Jr, Saad F, et al. 2011 Abiraterone and increased survival in metastatic prostate cancer. New England Journal of Medicine 364 1995-2005. (doi:10.1056/NEJMoa1014618)

De Laere B, van Dam PJ, Whitington T, Mayrhofer M, Diaz EH, Van den Eynden G, Vandebroek J, Del-Favero J, Van Laere S, Dirix L, et al. 2017 Comprehensive profiling of the androgen receptor in liquid biopsies from castration-resistant prostate cancer reveals novel Intra-AR structural variation and splice variant expression patterns. European Urology 72 192-200. (doi:10.1016/j.eururo.2017.01.011)

Dehm SM $2013 a$ mRNA splicing variants: exploiting modularity to outwit cancer therapy. Cancer Research 73 5309-5314. (doi:10.1158/0008-5472.CAN-13-0444)

Dehm SM $2013 b$ Test-firing ammunition for spliceosome inhibition in cancer. Clinical Cancer Research 19 6064-6066. (doi:10.1158/10780432.CCR-13-2461)

Dehm SM \& Tindall DJ 2007 Androgen receptor structural and functional elements: role and regulation in prostate cancer. Molecular Endocrinology 21 2855-2863. (doi:10.1210/me.2007-0223)

Dehm SM \& Tindall DJ 2011 Alternatively spliced androgen receptor variants. Endocrine-Related Cancer 18 R183-R196. (doi:10.1530/ERC11-0141)

Dehm SM, Schmidt LJ, Heemers HV, Vessella RL \& Tindall DJ 2008 Splicing of a novel androgen receptor exon generates a constitutively active androgen receptor that mediates prostate cancer therapy resistance. Cancer Research 68 5469-5477. (doi:10.1158/0008-5472. CAN-08-0594)

Feldman BJ \& Feldman D 2001 The development of androgenindependent prostate cancer. Nature Reviews Cancer $134-45$. (doi:10.1038/35094009)

Fizazi K, Scher HI, Miller K, Basch E, Sternberg CN, Cella D, Forer D, Hirmand M \& de Bono JS 2014 Effect of enzalutamide on time to first skeletal-related event, pain, and quality of life in men with castration-resistant prostate cancer: results from the randomised, phase 3 AFFIRM trial. Lancet Oncology 15 1147-1156. (doi:10.1016/ S1470-2045(14)70303-1)

Gong Y, Wang D, Dar JA, Singh P, Graham L, Liu W, Ai J, Xin Z, Guo Y \& Wang Z 2012 Nuclear export signal of androgen receptor (NESAR) regulation of androgen receptor level in human prostate cell lines via ubiquitination and proteasome-dependent degradation. Endocrinology 153 5716-5725. (doi:10.1210/en.2012-1841)

Guo Z, Yang X, Sun F, Jiang R, Linn DE, Chen H, Kong X, Melamed J, Tepper CG, Kung HJ, et al. 2009 A novel androgen receptor splice variant is up-regulated during prostate cancer progression and promotes androgen depletion-resistant growth. Cancer Research 69 2305-2313. (doi:10.1158/0008-5472.CAN-08-3795)

Han D, Gao S, Valencia K, Owiredu J, Han W, de Waal E, Macoska JA \& Cai C 2017 A novel nonsense mutation in androgen receptor confers resistance to CYP17 inhibitor treatment in prostate cancer. Oncotarget 8 6796-6808.

Henzler C, Li Y, Yang R, McBride T, Ho Y, Sprenger C, Liu G, Coleman I, Lakely B, Li R, et al. 2016 Truncation and constitutive activation of the androgen receptor by diverse genomic rearrangements in prostate cancer. Nature Communications 7 13668. (doi:10.1038/ ncomms13668)

Hornberg E, Ylitalo EB, Crnalic S, Antti H, Stattin P, Widmark A, Bergh A \& Wikstrom P 2011 Expression of androgen receptor splice variants in prostate cancer bone metastases is associated with castration-resistance and short survival. PLOS ONE 6 e19059. (doi:10.1371/journal.pone.0019059)

Hsu PD, Lander ES \& Zhang F 2014 Development and applications of CRISPR-Cas9 for genome engineering. Cell 157 1262-1278. (doi:10.1016/j.cell.2014.05.010)

Hu R, Dunn TA, Wei S, Isharwal S, Veltri RW, Humphreys E, Han M, Partin AW, Vessella RL, Isaacs WB, et al. 2009 Ligand-independent androgen receptor variants derived from splicing of cryptic exons signify hormone-refractory prostate cancer. Cancer Research 69 16-22. (doi:10.1158/0008-5472.CAN-08-2764)

Hu R, Isaacs WB \& Luo J 2011 A snapshot of the expression signature of androgen receptor splicing variants and their distinctive transcriptional activities. Prostate 71 1656-1667. (doi:10.1002/ pros.21382)

$\mathrm{Hu}$ R, Lu C, Mostaghel EA, Yegnasubramanian S, Gurel M, Tannahill C, Edwards J, Isaacs WB, Nelson PS, Bluemn E, et al. 2012 Distinct transcriptional programs mediated by the ligand-dependent fulllength androgen receptor and its splice variants in castrationresistant prostate cancer. Cancer Research 72 3457-3462. (doi:10.1158/0008-5472.CAN-11-3892)

Hu DG, McKinnon RA, Hulin JA, Mackenzie PI \& Meech R 2016 Novel nine-exon AR transcripts (Exon 1/Exon 1b/Exons 2-8) in normal and cancerous breast and prostate cells. International Journal of Molecular Sciences 18 pii E40. (doi:10.3390/ijms18010040)

Huggins C \& Hodges C 1941 Studies on prostate cancer: effect of castration, estrogen, and androgen injectionon serum phosphatases in metastatic carcinoma of the prostate. Cancer Research 1 293-297.

Isharwal S, Modi S, Arora N, Uhlrich C 3rd, Giri B, Barlass U, Soubra A, Chugh R, Dehm SM, Dudeja V, et al. 2017 Minnelide inhibits androgen dependent, castration resistant prostate cancer growth by decreasing expression of androgen receptor full length and splice variants. Prostate 77 584-596. (doi:10.1002/pros.23298)

Jagla M, Feve M, Kessler P, Lapouge G, Erdmann E, Serra S, Bergerat JP \& Ceraline J 2007 A splicing variant of the androgen receptor detected in a metastatic prostate cancer exhibits exclusively cytoplasmic actions. Endocrinology 148 4334-4343. (doi:10.1210/en.2007-0446)

Li Y, Chan SC, Brand LJ, Hwang TH, Silverstein KA \& Dehm SM 2013 Androgen receptor splice variants mediate enzalutamide resistance in castration-resistant prostate cancer cell lines. Cancer Research $\mathbf{7 3}$ 483-489. (doi:10.1158/0008-5472.CAN-12-3630)

Li H, Ban F, Dalal K, Leblanc E, Frewin K, Ma D, Adomat H, Rennie PS \& Cherkasov A 2014 Discovery of small-molecule inhibitors selectively targeting the DNA-binding domain of the human androgen receptor. Journal of Medicinal Chemistry 57 6458-6467. (doi:10.1021/ jm500802j)

Libertini SJ, Tepper CG, Rodriguez V, Asmuth DM, Kung HJ \& Mudryj M 2007 Evidence for calpain-mediated androgen receptor cleavage as

Published by Bioscientifica Ltd. 
a mechanism for androgen independence. Cancer Research 67 9001-9005. (doi:10.1158/0008-5472.CAN-07-1072)

Liu LL, Xie N, Sun S, Plymate S, Mostaghel E \& Dong X 2014 Mechanisms of the androgen receptor splicing in prostate cancer cells. Oncogene 33 3140-3150. (doi:10.1038/onc.2013.284)

Lu J, Lonergan PE, Nacusi LP, Wang L, Schmidt LJ, Sun Z, Van der Steen T, Boorjian SA, Kosari F, Vasmatzis G, et al. 2015 The cistrome and gene signature of androgen receptor splice variants in castration resistant prostate cancer cells. Journal of Urology 193 690-698. (doi:10.1016/j.juro.2014.08.043)

Liu C, Armstrong C, Zhu Y, Lou W \& Gao AC 2016 Niclosamide enhances abiraterone treatment via inhibition of androgen receptor variants in castration resistant prostate cancer. Oncotarget $\mathbf{7}$ 32210-32220.

Marcias G, Erdmann E, Lapouge G, Siebert C, Barthelemy P, Duclos B, Bergerat JP, Ceraline J \& Kurtz JE 2010 Identification of novel truncated androgen receptor (AR) mutants including unreported premRNA splicing variants in the 22Rv1 hormone-refractory prostate cancer (PCa) cell line. Human Mutation 31 74-80. (doi:10.1002/ humu.21138)

Miyamoto DT, Sequist LV \& Lee RJ 2014 Circulating tumour cellsmonitoring treatment response in prostate cancer. Nature Reviews Clinical Oncology 11 401-412. (doi:10.1038/nrclinonc.2014.82)

Miyamoto DT, Zheng Y, Wittner BS, Lee RJ, Zhu H, Broderick KT, Desai R, Fox DB, Brannigan BW, Trautwein J, et al. 2015 RNA-Seq of single prostate CTCs implicates noncanonical Wnt signaling in antiandrogen resistance. Science 349 1351-1356. (doi:10.1126/ science.aab0917)

Montgomery RB, Mostaghel EA, Vessella R, Hess DL, Kalhorn TF, Higano CS, True LD \& Nelson PS 2008 Maintenance of intratumoral androgens in metastatic prostate cancer: a mechanism for castrationresistant tumor growth. Cancer Research 68 4447-4454. (doi:10.1158/0008-5472.CAN-08-0249)

Mostaghel EA \& Plymate S 2011 New hormonal therapies for castrationresistant prostate cancer. Endocrinology and Metabolism Clinics of North America 40 625-642. (doi:10.1016/j.ecl.2011.05.013)

Mostaghel EA, Page ST, Lin DW, Fazli L, Coleman IM, True LD, Knudsen B, Hess DL, Nelson CC, Matsumoto AM, et al. 2007 Intraprostatic androgens and androgen-regulated gene expression persist after testosterone suppression: therapeutic implications for castrationresistant prostate cancer. Cancer Research 67 5033-5041. (doi:10.1158/0008-5472.CAN-06-3332)

Mostaghel EA, Marck BT, Plymate SR, Vessella RL, Balk S, Matsumoto AM, Nelson PS \& Montgomery RB 2011 Resistance to CYP17A1 inhibition with abiraterone in castration-resistant prostate cancer: induction of steroidogenesis and androgen receptor splice variants. Clinical Cancer Research 17 5913-5925. (doi:10.1158/1078-0432.CCR11-0728)

Mostaghel EA, Plymate SR \& Montgomery B 2014 Molecular pathways: targeting resistance in the androgen receptor for therapeutic benefit. Clinical Cancer Research 20 791-798. (doi:10.1158/1078-0432.CCR12-3601)

Myung JK, Banuelos CA, Fernandez JG, Mawji NR, Wang J, Tien AH, Yang YC, Tavakoli I, Haile S, Watt K, et al. 2013 An androgen receptor $\mathrm{N}$-terminal domain antagonist for treating prostate cancer. Journal of Clinical Investigation 123 2948-2960. (doi:10.1172/ JCI66398)

Nadiminty N \& Gao AC 2012 Mechanisms of persistent activation of the androgen receptor in CRPC: recent advances and future perspectives. World Journal of Urology 30 287-295. (doi:10.1007/ s00345-011-0771-3)

Nadiminty N, Tummala R, Liu C, Yang J, Lou W, Evans CP \& Gao AC 2013 NF-kappaB2/p52 induces resistance to enzalutamide in prostate cancer: role of androgen receptor and its variants. Molecular Cancer Therapeutics 12 1629-1637. (doi:10.1158/1535-7163.MCT-13-0027)
Nadiminty N, Tummala R, Liu C, Lou W, Evans CP \& Gao AC 2015 NF-kappaB2/p52:c-Myc:hnRNPA1 pathway regulates expression of androgen receptor splice variants and enzalutamide sensitivity in prostate cancer. Molecular Cancer Therapeutics 14 1884-1895. (doi:10.1158/1535-7163.MCT-14-1057)

Njar VC \& Brodie AM 2015 Discovery and development of Galeterone (TOK-001 or VN/124-1) for the treatment of all stages of prostate cancer. Journal of Medicinal Chemistry 58 2077-2087. (doi:10.1021/ jm501239f)

Nyquist MD \& Dehm SM 2013 Interplay between genomic alterations and androgen receptor signaling during prostate cancer development and progression. Hormone Cancer 4 61-69. (doi:10.1007/s12672-0130131-4)

Pelley RP, Chinnakannu K, Murthy S, Strickland FM, Menon M, Dou QP, Barrack ER \& Reddy GP 2006 Calmodulin-androgen receptor (AR) interaction: calcium-dependent, calpain-mediated breakdown of AR in LNCaP prostate cancer cells. Cancer Research 66 11754-11762. (doi:10.1158/0008-5472.CAN-06-2918)

Robinson D, Van Allen EM, Wu YM, Schultz N, Lonigro RJ, Mosquera JM, Montgomery B, Taplin ME, Pritchard CC, Attard G, et al. 2015 Integrative clinical genomics of advanced prostate cancer. Cell $\mathbf{1 6 1}$ 1215-1228. (doi:10.1016/j.cell.2015.05.001)

Romanel A, Gasi Tandefelt D, Conteduca V, Jayaram A, Casiraghi N, Wetterskog D, Salvi S, Amadori D, Zafeiriou Z, Rescigno P, et al. 2015 Plasma AR and abiraterone-resistant prostate cancer. Science Translational Medicine 7 312re310.

Saporita AJ, Zhang Q, Navai N, Dincer Z, Hahn J, Cai X \& Wang Z 2003 Identification and characterization of a ligand-regulated nuclear export signal in androgen receptor. Journal of Biological Chemistry 278 41998-42005. (doi:10.1074/jbc.M302460200)

Sartor O \& Dong Y 2015 Androgen receptor variant-7: an important predictive biomarker in castrate resistant prostate cancer. Asian Journal of Andrology 17 439-440.

Scher HI, Lu D, Schreiber NA, Louw J, Graf RP, Vargas HA, Johnson A, Jendrisak A, Bambury R, Danila D, et al. 2016 Association of AR-V7 on circulating tumor cells as a treatment-specific biomarker with outcomes and survival in castration-resistant prostate cancer. JAMA Oncology 2 1441-1449. (doi:10.1001/jamaoncol.2016.1828)

Sharifi N 2015a Prostate cancer: CYP17A1 inhibitor failure-lessons for future drug development. Nature Reviews Urology 12 245-246. (doi:10.1038/nrurol.2015.66)

Sharifi N 2015b Steroid sidestep: evading androgen ablation by abiraterone. Clinical Cancer Research 21 1240-1242. (doi:10.1158/1078-0432.CCR-14-2899)

Sharifi N, Gulley JL \& Dahut WL 2010 An update on androgen deprivation therapy for prostate cancer. Endocrine-Related Cancer $\mathbf{1 7}$ R305-R315. (doi:10.1677/ERC-10-0187)

Sprenger CC \& Plymate SR 2014 The link between androgen receptor splice variants and castration-resistant prostate cancer. Hormones and Cancer 5 207-217.

Sun S, Sprenger CC, Vessella RL, Haugk K, Soriano K, Mostaghel EA, Page ST, Coleman IM, Nguyen HM, Sun H, et al. 2010 Castration resistance in human prostate cancer is conferred by a frequently occurring androgen receptor splice variant. Journal of Clinical Investigation 120 2715-2730. (doi:10.1172/JCI41824)

Taplin ME, Montgomery B, Logothetis CJ, Bubley GJ, Richie JP, Dalkin BL, Sanda MG, Davis JW, Loda M, True LD, et al. 2014 Intense androgen-deprivation therapy with abiraterone acetate plus leuprolide acetate in patients with localized high-risk prostate cancer: results of a randomized phase II neoadjuvant study. Journal of Clinical Oncology 32 3705-3715. (doi:10.1200/JCO.2013.53.4578)

Thadani-Mulero M, Portella L, Sun S, Sung M, Matov A, Vessella RL, Corey E, Nanus DM, Plymate SR \& Giannakakou P 2014 Androgen receptor splice variants determine taxane sensitivity in prostate cancer. Cancer Research 74 2270-2282. (doi:10.1158/0008-5472.CAN-13-2876) http://erc.endocrinology-journals.org

DOI: 10.1530/ERC-17-0108
(C) 2017 Society for Endocrinology Printed in Great Britain
Published by Bioscientifica Ltd 
Uo T, Dvinge H, Sprenger CC, Bradley RK, Nelson PS \& Plymate SR 2017 Systematic and functional characterization of novel androgen receptor variants arising from alternative splicing in the ligandbinding domain. Oncogene 36 1440-1450. (doi:10.1038/ onc.2016.313)

Wadosky KM \& Koochekpour S 2017 Androgen receptor splice variants and prostate cancer: from bench to bedside. Oncotarget $\mathbf{8}$ 18550-18576.

Wang QM, Fan GC, Chen JZ, Chen HP \& He FC 2004 A putative NES mediates cytoplasmic localization of Apoptin in normal cells. Acta Biochimica et Biophysica Sinica 36 817-823. (doi:10.1093/ abbs/36.12.817)

Wang J, Zou JX, Xue X, Cai D, Zhang Y, Duan Z, Xiang Q, Yang JC, Louie MC, Borowsky AD, et al. 2016a ROR-gamma drives androgen receptor expression and represents a therapeutic target in castrationresistant prostate cancer. Nature Medicine 22 488-496. (doi:10.1038/ nm.4070)

Wang R, Lin W, Lin C, Li L, Sun Y \& Chang C 2016b ASC-J9((R)) suppresses castration resistant prostate cancer progression via degrading the enzalutamide-induced androgen receptor mutant AR-F876L. Cancer Letters 379 154-160. (doi:10.1016/j.canlet.2016.05.018)

Ware KE, Garcia-Blanco MA, Armstrong AJ \& Dehm SM 2014 Biologic and clinical significance of androgen receptor variants in castration resistant prostate cancer. Endocrine-Related Cancer 21 T87-T103. (doi:10.1530/ERC-13-0470)

Watson PA, Chen YF, Balbas MD, Wongvipat J, Socci ND, Viale A, Kim K \& Sawyers CL 2010 Constitutively active androgen receptor splice variants expressed in castration-resistant prostate cancer require fulllength androgen receptor. PNAS 107 16759-16765. (doi:10.1073/ pnas.1012443107)

Welti J, Rodrigues DN, Sharp A, Sun S, Lorente D, Riisnaes R, Figueiredo I, Zafeiriou Z, Rescigno P, de Bono JS, et al. 2016 Analytical validation and clinical qualification of a new immunohistochemical assay for androgen receptor splice variant-7 protein expression in metastatic castration-resistant prostate cancer. European Urology 70 599-608. (doi:10.1016/j.eururo.2016.03.049)

Wen S, Tian J, Niu Y, Li L, Yeh S \& Chang C 2016 ASC-J9((R)), and not casodex or enzalutamide, suppresses prostate cancer stem/progenitor cell invasion via altering the EZH2-STAT3 signals. Cancer Letters $\mathbf{3 7 6}$ 377-386. (doi:10.1016/j.canlet.2016.01.057)

Xu D, Zhan Y, Qi Y, Cao B, Bai S, Xu W, Gambhir SS, Lee P, Sartor O, Flemington EK, et al. 2015 Androgen receptor splice variants dimerize to transactivate target genes. Cancer Research 75 3663-3671. (doi:10.1158/0008-5472.CAN-15-0381)

Yamashita S, Lai KP, Chuang KL, Xu D, Miyamoto H, Tochigi T, Pang ST, Li L, Arai Y, Kung HJ, et al. 2012 ASC-J9 suppresses castrationresistant prostate cancer growth through degradation of full-length and splice variant androgen receptors. Neoplasia 14 74-83. (doi:10.1593/neo.111436)

Yang H, Murthy S, Sarkar FH, Sheng S, Reddy GP \& Dou QP 2008 Calpain-mediated androgen receptor breakdown in apoptotic prostate cancer cells. Journal of Cellular Physiology 217 569-576. (doi:10.1002/jcp.21565)

Yang X, Guo Z, Sun F, Li W, Alfano A, Shimelis H, Chen M, Brodie AM, Chen H, Xiao Z, et al. 2011 Novel membrane-associated androgen receptor splice variant potentiates proliferative and survival responses in prostate cancer cells Journal of Biological Chemistry 286 36152-36160. (doi:10.1074/jbc.M111.265124)

Yang YC, Banuelos CA, Mawji NR, Wang J, Kato M, Haile S, McEwan IJ, Plymate S \& Sadar MD 2016 Targeting androgen receptor activation function-1 with epi to overcome resistance mechanisms in castration-resistant prostate cancer. Clinical Cancer Research 22 4466-4477. (doi:10.1158/1078-0432.CCR-15-2901)

Zhan Y, Zhang G, Wang X, Qi Y, Bai S, Li D, Ma T, Sartor O, Flemington EK, Zhang H, et al. 2017 Interplay between cytoplasmic and nuclear androgen receptor splice variants mediates castration resistance. Molecular Cancer Research 15 59-68. (doi:10.1158/1541-7786.MCR16-0236)

Received in final form 19 June 2017

Accepted 13 July 2017
() 2017 Society for Endocrinology Printed in Great Britain
Published by Bioscientifica Ltd 